\title{
Supplementary Information for Colloidal Quantum Dot Solar Cell Band Alignment using Two-Step Ionic Doping
}

Koen Bertens ${ }^{\S}$, James Z. Fan ${ }^{\S}$, Margherita Biondi ${ }^{\S}$, Armin Sedighian Rasouli ${ }^{\S}$, Seungjin Lee ${ }^{\S}$ Peicheng $\mathrm{Li}^{\dagger}$, Bin Sun ${ }^{\S}$, Sjoerd Hoogland», F. Pelayo García de Arquer», Zheng-Hong Luं, Edward H. Sargent ${ }^{\S *}$

\section{Experimental Methods}

\section{CQD Synthesis and Ligand Exchange}

Lead sulfide $(\mathrm{PbS})$ colloidal quantum dots (CQDs) were synthesized according to previous reports ${ }^{1}$. An exchange solution was prepared of $0.1 \mathrm{M} \mathrm{PbI}_{2}$ (99.999\%, Alfa Aesar), $0.04 \mathrm{M} \mathrm{PbBr}_{2}$ (98\%, Sigma-Aldrich), and 0.04 $\mathrm{M} \mathrm{NH}_{4} \mathrm{Ac}$ (99.999\%, Sigma-Aldrich) in $20 \mathrm{~mL}$ of N,Ndimethylformamide (DMF) (Anhydrous, 98\% Sigma-Aldrich). 20 mL of octane (98\%, Alfa Aesar) was added to the $20 \mathrm{~mL}$ exchange solution, and $2.8 \mathrm{~mL}$ of $950 \mathrm{~nm}$ oleic acid capped PbS CQDs were added to the octane phase. The mixture was vortexed for 90 seconds to transfer the CQDs to the DMF phase, and the octane phase was discarded. After this phase transfer, the new solution was washed three times by adding $20 \mathrm{~mL}$ of octane, vortexing for 30 seconds, and discarding the top octane phase. After the third wash, the CQD solution was transferred to four glass test tubes ( $5 \mathrm{~mL}$ per test tube) and the CQDs were precipitated with $3 \mathrm{~mL}$ toluene (distilled in glass, Caledon). The test tubes were centrifuged for two minutes to settle them to the bottom of the tube, and the supernatant was discarded. The CQDs were dried under vacuum for 14 minutes and dispersed in butylamine (99\%, Sigma-Aldrich) to a concentration of $300 \mathrm{mg} / \mathrm{mL}$ to form an ink. 
The ink was vortexed and centrifuged for 30 seconds at 13000 RPM. The supernatant was used for solar cell fabrication.

\section{CQD Fluoride Ion Doping}

A solution of $5 \mathrm{mM}, 10 \mathrm{mM}$, and $20 \mathrm{mM} \mathrm{SnF}_{2}$ (99\%, Sigma-Aldrich) was prepared in DMF. PbS CQD synthesis and ligand exchange was performed through standard procedure. After drying of exchanged CQDs, the CQDs were dispersed in $90 \%$ butylamine and $10 \% \mathrm{SnF}_{2}$ solution to a concentration of $300 \mathrm{mg} / \mathrm{mL}$. The dispersion was vortexed and centrifuged for 30 seconds at 13000 RPM. The supernatant of the dispersion was used for solar cell fabrication.

\section{$\underline{\text { CQD Solar Cell Fabrication }}$}

A patterned indium tin oxide (ITO)-coated glass substrate was used for fabrication of the solar cell. Two layers of $\mathrm{ZnO}$ nanoparticle solution were spin coated on top of the cleaned ITO substrate at 5000 RPM for 30 seconds. A CQD film was spin coated on top by injecting 40 to $50 \mu \mathrm{L}$ ink supernatant onto the substrate and spin coating for 30 seconds at 1500 to 2000 RPM. The films were annealed at $70^{\circ} \mathrm{C}$ for 15 minutes in a $\mathrm{N}_{2}$ filled glovebox. A solution of $6 \mathrm{mg} / \mathrm{mL} \mathrm{FABr}$ in acetonitrile (ACN) was prepared. The annealed films were spun at 2500 RMP for 20 seconds, and $0.7 \mathrm{~mL}$ of $\mathrm{FABr}$ solution was injected as per previous report ${ }^{2}$. The films were again annealed at $70^{\circ} \mathrm{C}$ for 10 minutes in a glovebox, and after annealing, the films were washed with ACN by drop casting, followed by a final annealing step at $70^{\circ} \mathrm{C}$ for 5 minutes in glovebox. A 1,2-ethanedithiol (EDT)-PbS hole transport layer was deposited on top through a layer-by-layer ligand exchange; $50 \mu \mathrm{L}$ of $850 \mathrm{~nm}$ oleic acid capped CQDs in $50 \mathrm{mg} / \mathrm{mL}$ octane solution were spin coated for 10 seconds at 2500 RPM, followed by a 10 second soaking of $0.01 \%$ EDT solution in 3:2 ACN:ethyl acetate (EA), followed by three washes of the same ACN and EA mixture. This process was 
repeated for a total of two times. A $120 \mathrm{~nm}$ layer of Au was deposited as the top electrode by electron beam evaporation.

\section{$\underline{\text { AM1.5G Solar Efficiency Measurement }}$}

A $0.049 \mathrm{~cm}^{2}$ aperture was used to illuminate the pixel with an AM1.5G solar simulator (Sciencetech class A). The light intensity was calibrated to a brightness of 1 sun using a NISTtraceable calibrated reference cell (Newport $91150 \mathrm{~V}$ ). A continuous nitrogen gas flow was used during testing of the solar cell. Current density-voltage measurements were recorded using a multimeter (Keithley 2400). A scanning voltage range of $-0.7 \mathrm{~V}$ to $0.1 \mathrm{~V}$ was used.

\section{$\underline{\text { External Quantum Efficiency Measurement }}$}

External quantum efficiency (EQE) spectra were measured using a Newport Quant-X 300 measurement system. The solar cell was measured under continuous white light illumination. A spectral range of $350 \mathrm{~nm}$ to $1200 \mathrm{~nm}$ was used. EQE corrected $\mathrm{J}_{\mathrm{SC}}$ was acquired from the measurement and used to calculate an EQE corrected PCE according to the following formula: $\mathrm{PCE}=\mathrm{V}_{\mathrm{OC}} * \mathrm{~J}_{\mathrm{SC}} * \mathrm{FF}$

\section{Ultraviolet Photoelectron Spectroscopy Measurements}

A helium discharge source $(\mathrm{HeI} \alpha, \mathrm{hv}=21.22 \mathrm{eV})$ was used and samples were placed at a take-off angle of $88^{\circ}$. The sample was held at a $-15 \mathrm{~V}$ bias relative to the spectrometer in order to efficiently collect low kinetic-energy electrons. The Fermi level energy was calculated from the equation: EF 
$=21.22 \mathrm{eV}-\mathrm{SEC}$, where SEC is the secondary electron cut-off. The difference between valence band $(\mathrm{VB})$ and $\mathrm{EF}, \eta$, was determined from the $\mathrm{VB}$ onset in the $\mathrm{VB}$ region.

$\underline{\text { X-ray Photoelectron Spectroscopy Measurements }}$

CQD active layers were prepared using the same methodology as for solar cell fabrication, directly onto an ITO substrate. Films were mounted onto a stainless-steel mounting plate and measurements were taken with a Thermo Scientific K-Alpha system with Al Ka source. The takeoff angle used was $90^{\circ}$. The XPS spectra were calibrated to the C 1s peak at $284.8 \mathrm{eV}$.

\section{$\underline{\text { SCAPS Simulations }}$}

SCAPS simulation software was used to construct and simulate the figures of merit of a CQD solar cell. To explore the effect of doping, the electron affinity value was swept from $3.85 \mathrm{eV}$ to 4.15 $\mathrm{eV}$. The shallow uniform acceptor density was varied from $10^{14} \mathrm{~cm}^{-3}$ to $10^{17} \mathrm{~cm}^{-3}$. Further information can be found in Supplementary Table S3.

\section{Supplementary Figures}



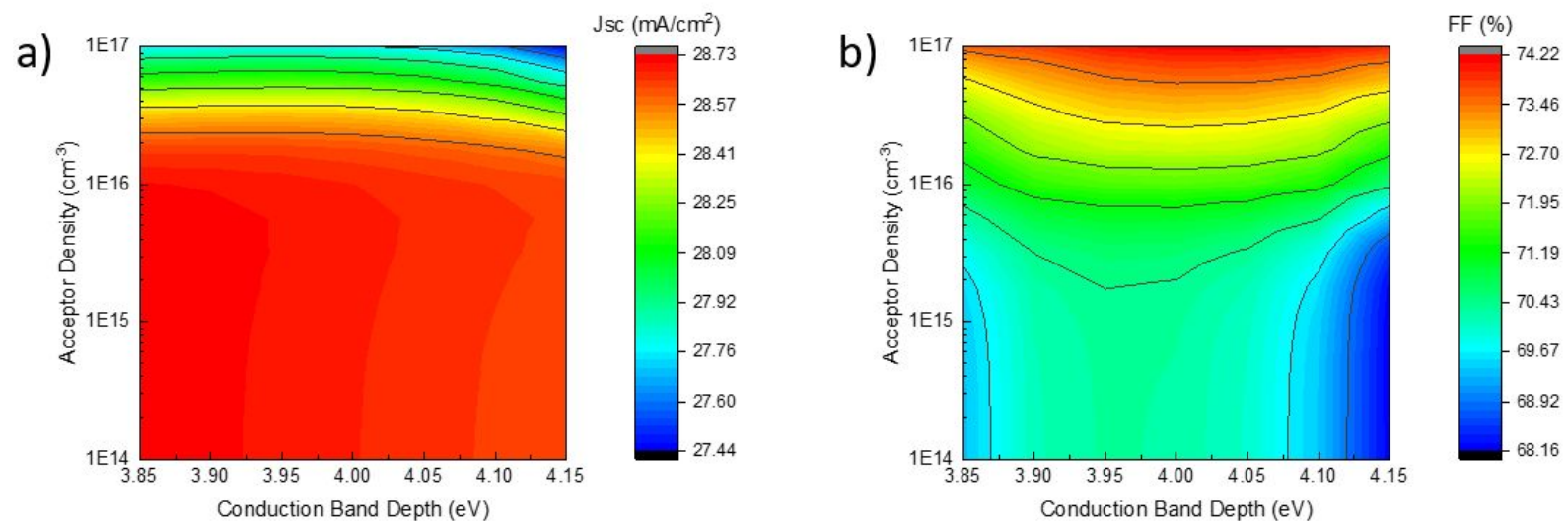

Figure S1. Simulated short-circuit current density (a) and fill factor (b) of a CQD solar cell with variable conduction band depth and acceptor density.
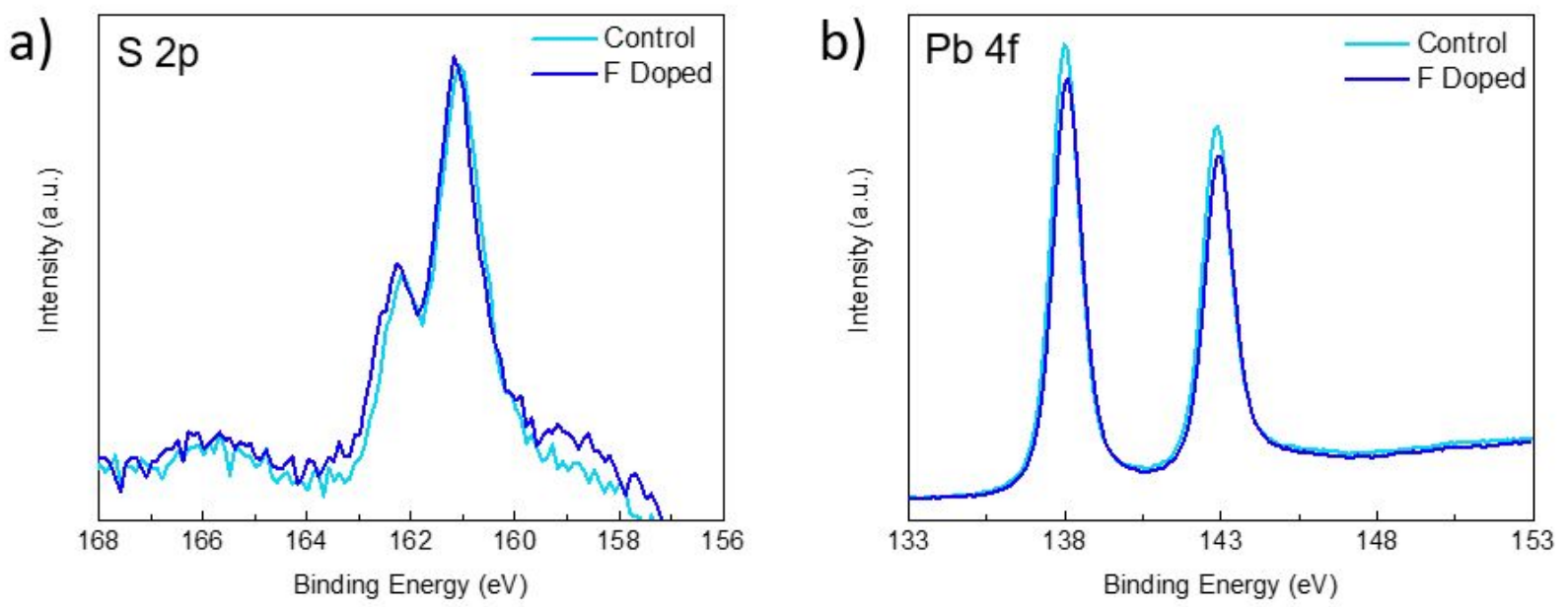

Figure S2. XPS spectra of S $2 p(a)$ and $\mathrm{Pb} 4 \mathrm{f}(\mathrm{b})$. 

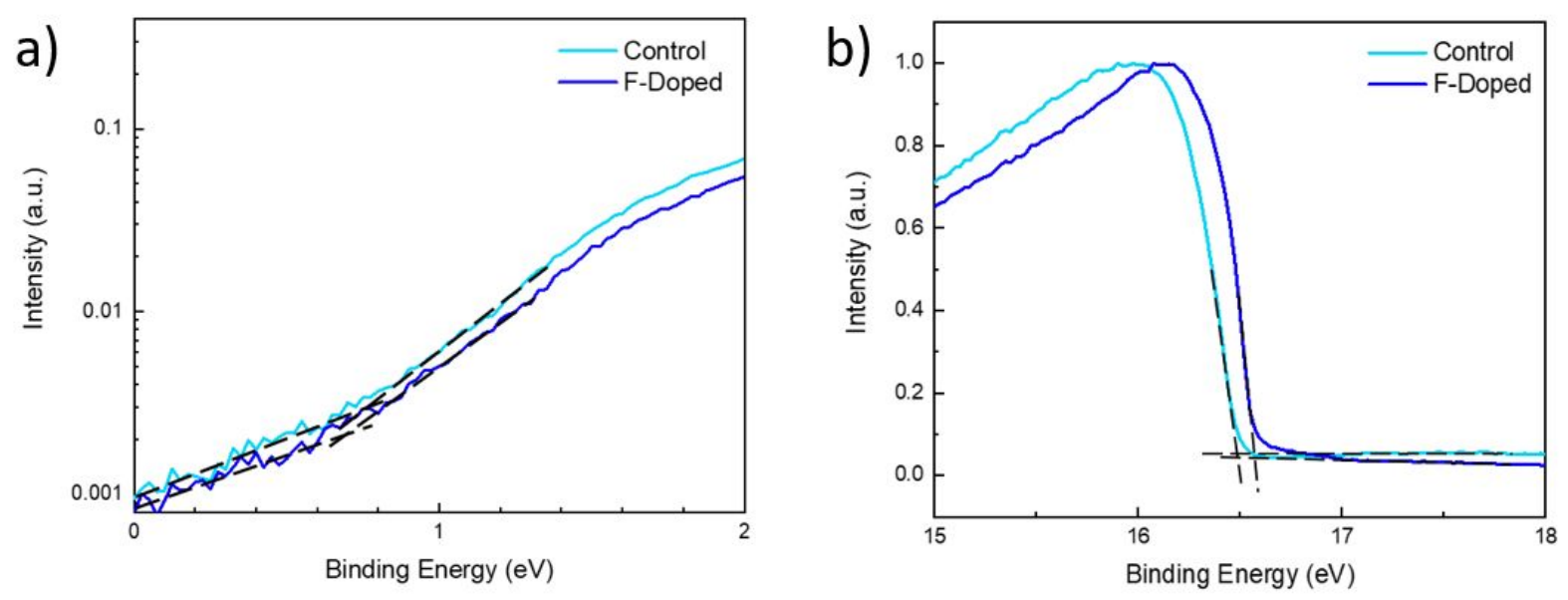

Figure S3. UPS spectra of control and fluoride doped CQD films showing semi-logarithmic valence band regions (a) and secondary cut-off regions (b).

Table S1. Solar cell figures of merit under full spectrum illumination for control and fluoridebased exchange CQD devices.

\begin{tabular}{|l|l|l|l|l|}
\hline Sample & $\mathbf{V}_{\text {OC }}(\mathbf{V})$ & $\mathbf{J}_{\text {SC }}\left(\mathbf{m A} / \mathbf{c m}^{2}\right)$ & FF (\%) & PCE (\%) \\
\hline Control & 0.644 & 28.1 & 64.5 & 11.7 \\
\hline $\begin{array}{l}\text { Fluoride } \\
\text { Exchange }\end{array}$ & 0.647 & 27.8 & 57.1 & 10.3 \\
\hline
\end{tabular}

Table S2. Solar cell figures of merit average values and standard deviation under full spectrum illumination for control and fluoride doped CQD devices.

\begin{tabular}{|l|l|l|l|l|l|l|}
\hline Sample & $\mathbf{V}_{\text {OC }}(\mathbf{V )}$ & $\begin{array}{l}\mathbf{J}_{\mathbf{S C}} \\
\left(\mathbf{m A} / \mathbf{c m}^{\mathbf{2}}\right)\end{array}$ & FF (\%) & PCE (\%) & $\begin{array}{l}\text { Champion } \\
\text { PCE (\%) }\end{array}$ & $\begin{array}{l}\text { Thickness } \\
(\mathbf{n m})\end{array}$ \\
\hline Control & $0.643 \pm 0.004$ & $27.3 \pm 0.6$ & $64.3 \pm 1.7$ & $11.3 \pm 0.3$ & 12.0 & 372 \\
\hline $5 \mathrm{mM}$ & $0.651 \pm 0.002$ & $27.9 \pm 0.8$ & $66.3 \pm 1.6$ & $12.0 \pm 0.3$ & 12.6 & 301 \\
\hline $10 \mathrm{mM}$ & $0.649 \pm 0.002$ & $28.0 \pm 0.6$ & $66.3 \pm 1.9$ & $12.1 \pm 0.3$ & 12.7 & 352 \\
\hline $20 \mathrm{mM}$ & $0.642 \pm 0.002$ & $27.4 \pm 0.7$ & $63.5 \pm 2.1$ & $11.2 \pm 0.2$ & 11.6 & 321 \\
\hline
\end{tabular}

Table S3. SCAPS simulation parameters based on literature ${ }^{2,3}$.

\begin{tabular}{|l|c|c|c|}
\hline \multirow{2}{*}{$\mathrm{ZnO}$} & \multicolumn{3}{|c|}{ Active Layer - EDT-PbS } \\
\cline { 3 - 4 } & Halide-PbS & EDT-PbS & $\begin{array}{c}\text { Composition } \\
\text { Dependence }\end{array}$ \\
\hline
\end{tabular}




\begin{tabular}{|c|c|c|c|c|}
\hline Thickness (nm) & 150 & \multicolumn{2}{|c|}{450} & \\
\hline Bandgap (eV) & 3.50 & 1.27 & 1.40 & Linear \\
\hline $\begin{array}{l}\text { Electron affinity } \\
(\mathrm{eV})\end{array}$ & 4.10 & Varied & 3.80 & Linear \\
\hline $\begin{array}{l}\text { Dielectric } \\
\text { permittivity }\end{array}$ & 66.0 & 20.0 & 20.0 & Linear \\
\hline $\begin{array}{l}\text { CB effective } \\
\text { density of states } \\
\left(\mathrm{cm}^{-3}\right)\end{array}$ & 1E19 & $1 \mathrm{E} 21$ & $1 \mathrm{E} 21$ & Linear \\
\hline $\begin{array}{l}\text { VB effective } \\
\text { density of states } \\
\left(\mathrm{cm}^{-3}\right)\end{array}$ & $1 \mathrm{E} 19$ & $1 \mathrm{E} 21$ & $1 \mathrm{E} 21$ & Linear \\
\hline $\begin{array}{l}\text { Electron thermal } \\
\text { velocity }(\mathrm{cm} / \mathrm{s})\end{array}$ & $1 \mathrm{E} 7$ & $3.09 \mathrm{E} 4$ & $3.09 \mathrm{E} 4$ & Linear \\
\hline $\begin{array}{l}\text { Hole thermal } \\
\text { velocity }(\mathrm{cm} / \mathrm{s})\end{array}$ & $1 \mathrm{E} 7$ & $3.09 \mathrm{E} 4$ & $3.09 \mathrm{E} 4$ & Linear \\
\hline $\begin{array}{l}\text { Electron mobility } \\
\left(\mathrm{cm}^{2} / \mathrm{Vs}\right)\end{array}$ & $5 \mathrm{E}-1$ & $2 \mathrm{E}-1$ & $8 \mathrm{E}-2$ & Linear \\
\hline $\begin{array}{l}\text { Hole mobility } \\
\left(\mathrm{cm}^{2} / \mathrm{Vs}\right)\end{array}$ & $5 \mathrm{E}-1$ & $2 \mathrm{E}-1$ & $8 \mathrm{E}-2$ & Linear \\
\hline $\begin{array}{l}\text { Shallow donor } \\
\text { density }\left(\mathrm{cm}^{-3}\right)\end{array}$ & 1E18 & 1E15 & 0 & Linear \\
\hline $\begin{array}{l}\text { Shallow acceptor } \\
\text { density }\left(\mathrm{cm}^{-3}\right)\end{array}$ & 0 & Varied & 1E18 & Linear \\
\hline
\end{tabular}

\section{References}

(1) Ning, Z.; Voznyy, O.; Pan, J.; Hoogland, S.; Adinolfi, V.; Xu, J.; Li, M.; Kirmani, A. R.;

Sun, J. P.; Minor, J.; Kemp, K. W.; Dong, H.; Rollny, L.; Labelle, A.; Carey, G.;

Sutherland, B.; Hill, I.; Amassian, A.; Liu, H.; Tang, J.; Bakr, O. M.; Sargent, E. H. Air-

Stable n-Type Colloidal Quantum Dot Solids. Nat. Mater. 2014, 13 (8), 822-828.

https://doi.org/10.1038/nmat4007.

(2) Sun, B.; Johnston, A.; Xu, C.; Wei, M.; Huang, Z.; Jiang, Z.; Zhou, H.; Gao, Y.; Dong, Y.; Ouellette, O.; Zheng, X.; Liu, J.; Choi, M.-J.; Gao, Y.; Baek, S.-W.; Laquai, F.; Bakr, O. M.; Ban, D.; Voznyy, O.; García de Arquer, F. P.; Sargent, E. H. Monolayer Perovskite Bridges Enable Strong Quantum Dot Coupling for Efficient Solar Cells. Joule 2020, 1-15. 
https://doi.org/10.1016/j.joule.2020.05.011.

(3) Biondi, M.; Choi, M. J.; Ouellette, O.; Baek, S. W.; Todorović, P.; Sun, B.; Lee, S.; Wei, M.; Li, P.; Kirmani, A. R.; Sagar, L. K.; Richter, L. J.; Hoogland, S.; Lu, Z. H.; García de Arquer, F. P.; Sargent, E. H. A Chemically Orthogonal Hole Transport Layer for Efficient Colloidal Quantum Dot Solar Cells. Adv. Mater. 2020, 32 (17), 1-8.

https://doi.org/10.1002/adma.201906199. 